\title{
A Study on Answering a Data Mining Query Using a Materialized View*
}

\author{
Maciej Zakrzewicz, Mikolaj Morzy, Marek Wojciechowski \\ Poznan University of Technology \\ Institute of Computing Science \\ ul. Piotrowo 3a, 60-965 Poznan, Poland \\ $\{$ mzakrz, mmorzy, marek\} @cs.put.poznan.pl
}

\begin{abstract}
One of the classic data mining problems is discovery of frequent itemsets. This problem particularly attracts database community as it resembles traditional database querying. In this paper we consider a data mining system which supports storing of previous query results in the form of materialized data mining views. While numerous works have shown that reusing results of previous frequent itemset queries can significantly improve performance of data mining query processing, a thorough study of possible differences between the current query and a materialized view has not been presented yet. In this paper we classify possible differences into six classes, provide $\mathrm{I} / \mathrm{O}$ cost analysis for all the classes, and experimentally evaluate the most promising ones.
\end{abstract}

\section{Introduction}

Data mining aims at discovery of useful patterns from large databases or warehouses. Nowadays we are witnessing the evolution of data mining environments from specialized tools to multi-purpose data mining systems offering some level of integration with existing database management systems. Data mining can be seen as advanced querying, where a user specifies the source dataset and the requested pattern constraints, then the system chooses the appropriate data mining algorithm and returns the discovered patterns to the user. Data mining query processing has recently become an important research area focusing mainly on constraint handling and reusing results of previous queries.

In our previous work we introduced the concept of materialized data mining views, providing a general discussion on their possible usage in mining various classes of frequent patterns [8][9]. In this paper we focus on the most prominent class of patterns - frequent itemsets. We present a thorough study of possible differences between the current frequent itemset query and a materialized view. We identify six classes of possible differences, providing I/O cost analysis for each of them. For the most promising classes we report results of conducted experiments.

\footnotetext{
* This work was partially supported by the grant no. 4T11C01923 from the State Committee for Scientific Research (KBN), Poland.
} 
discover frequent itemsets in an incremented dataset, exploiting previously discovered frequent itemsets.

The notion of data mining queries (or $K D D$ queries) was introduced in [7]. The need for Knowledge and Data Management Systems (KDDMS) as second generation data mining tools was expressed. The ideas of application programming interfaces and data mining query optimizers were also mentioned.

In [10] the authors postulated to create a knowledge cache that would keep recently discovered frequent itemsets along with their support value, in order to facilitate interactive and iterative mining. Besides presenting the notion of knowledge cache the authors introduced several maintenance techniques for such cache, and discussed using the cache contents when answering new frequent set queries.

In [4] three relationships which occur between two association rule queries were identified. The relationships represented cases when results on one query can be used to efficiently answer the other. However, the relationships concerned association rules - not frequent itemsets.

The work on materialized views started in the 80s. The basic concept was to use materialized views as a tool to speed up queries and serve older copies of data. Materialized views have become a key element of data warehousing technology (see [11] for an overview).

\section{Basic Definitions and Problem Formulation}

Definition 1 (Data mining query). A data mining query for frequent itemset discovery is a tuple $d m q=(\mathbb{R}, a, \Sigma, \Phi, \beta)$, where $R$ is a database relation, $a$ is a setvalued attribute of $R, \Sigma$ is a data selection predicate on $R, \Phi$ is a selection predicate on frequent itemsets, $\beta$ is the minimum support for the frequent itemsets. The data mining query $d m q$ returns all frequent itemsets discovered in $\pi_{a} \sigma_{\Sigma} R_{0}$ having support greater than $\beta$ and satisfying the constraints $\Phi$.

Example. Given is the database relation $\mathcal{R}_{I}\left(a t t r 1, a t t r_{2}\right)$. The data mining query $d m q_{1}$ $=\left(\mathcal{R}_{1}\right.$, "attr2", "attr $1>5$ ", "|itemset $\mid<4$ ", 3) describes the problem of discovering frequent itemsets in the set-valued attribute attr 2 of the relation $\mathcal{R}_{1}$. The frequent itemsets with support above 3 and length less than 4 are discovered in records having attri $>5$.

Definition 2 (Materialized data mining view). A materialized data mining view $d m v=(\mathbb{R} a, \Sigma, \Phi, \beta)$ is a data mining query, whose both the definition and the result are permanently stored (materialized) in a database. All frequent itemsets being a result of the data mining query are called materialized data mining view contents.

Definition 3 (Restricted frequent itemset selection predicate). Given are two data mining queries: $d m q_{l}=\left(\mathbb{R} a, \Sigma_{1}, \Phi_{1}, \beta_{l}\right)$ i $d m q_{2}=\left(R_{2} a, \Sigma_{2}, \Phi_{2}, \beta_{2}\right)$. We say that the frequent itemset selection predicate $\Phi_{l}$ is restricted with respect to the frequent itemset 
selection predicate $\Phi_{2}$ (or $\Phi_{2}$ is relaxed with respect to $\Phi_{1}$ ), written as $\Phi_{2} \subset \Phi_{1}$, if and only if for each frequent itemset, satisfying $\Phi_{1}$ implies also satisfying $\Phi_{2}$. We say that the frequent itemset selection predicates are independent if $\Phi_{1} \not \subset \Phi_{2} \wedge \Phi_{2} \not \subset \Phi_{1} \wedge \Phi_{1} \neq \Phi_{2}$.

Definition 4 (Stronger frequent itemset selection predicate). Given are two selection predicates on frequent itemsets: $p_{1}$ i $p_{2}$. We say that $p_{1}$ is stronger than $p_{2}$ if any of the conditions shown in Table 1 holds (We assume that items are integers. $S$ represents a frequent itemset, $\min () / \max ()$ returns the highest/lowest item, $\operatorname{count}()$ returns the size of an itemset, $\operatorname{sum}()$ returns the sum of all items, range() returns the difference between the highest and the lowest item, $V_{l}$ and $V_{2}$ are sets of items, $v_{l}$ and $v_{2}$ are integers).

Table 1. Conditions for $p_{1}$ being stronger than $p_{2}$

\begin{tabular}{|l|l|l|}
\hline \multicolumn{1}{|c|}{$p_{1}$} & \multicolumn{1}{|c|}{$p_{2}$} & condition \\
\hline$S \supseteq V_{1}$ & $S \supseteq V_{2}$ & $V_{I} \supset V_{2}$ \\
\hline$S \subseteq V_{1}$ & $S \subseteq V_{2}$ & $V_{l} \subset V_{2}$ \\
\hline $\min (S) \leq v_{1}$ & $\min (S) \leq v_{2}$ & $v_{1}<v_{2}$ \\
\hline $\min (S) \geq v_{1}$ & $\min (S) \geq v_{2}$ & $v_{1}>v_{2}$ \\
\hline $\max (S) \leq v_{1}$ & $\max (S) \leq v_{2}$ & $v_{1}<v_{2}$ \\
\hline $\max (S) \geq v_{1}$ & $\max (S) \geq v_{2}$ & $v_{1}>v_{2}$ \\
\hline $\operatorname{count}(S) \leq v_{1}$ & $\operatorname{count}(S) \leq v_{2}$ & $v_{1}<v_{2}$ \\
\hline $\operatorname{count}(S) \geq v_{2}$ & $\operatorname{count}(S) \geq v_{2}$ & $v_{1}>v_{2}$ \\
\hline $\operatorname{sum}(S) \leq v_{1}(\forall x \in S, a \geq 0)$ & $\operatorname{sum}(S) \leq v_{2}(\forall x \in S, a \geq 0)$ & $v_{1}<v_{2}$ \\
\hline $\operatorname{sum}(S) \geq v_{1}(\forall x \in S, a \geq 0)$ & $\operatorname{sum}(S) \geq v_{2}(\forall x \in S, a \geq 0)$ & $v_{1}>v_{2}$ \\
\hline $\operatorname{range}(S) \leq v_{1}$ & $\operatorname{range}(S) \leq v_{2}$ & $v_{1}<v_{2}$ \\
\hline $\operatorname{range}(S) \geq v_{1}$ & $\operatorname{range}(S) \geq v_{2}$ & $v_{1}>v_{2}$ \\
\hline
\end{tabular}

Theorem 1. Given are two data mining queries: $d m q_{l}=\left(\mathcal{R}_{2} a, \Sigma_{l}, \Phi_{l}, \beta_{l}\right)$ and $d m q_{2}=\left(R_{2} a, \Sigma_{2}, \Phi_{2}, \beta_{2}\right)$. The frequent itemset selection predicate $\Phi_{1}$ is restricted with respect to the frequent itemset selection predicate $\Phi_{2}$ if any of the following holds:

(1) The selection predicate $\Phi_{2}$ is a conjunction of $n$ predicates $p_{1}{ }^{2} \wedge p_{2}{ }^{2} \wedge \ldots \wedge p_{n}{ }^{2}$, the selection predicate $\Phi_{l}$ is a conjunction of $n+1$ predicates $p_{1}{ }^{l} \wedge p_{2}{ }^{l} \wedge \ldots \wedge p_{n}{ }^{l} \wedge p_{n+1}{ }^{l}$, and for each $1 \leq i \leq n$ we have $p_{i}{ }^{l}=p_{i}^{2}$.

(2) The selection predicate $\Phi_{1}$ is a conjunction of $n$ predicates $p_{1}{ }^{I} \wedge p_{2}{ }^{I} \wedge \ldots \wedge p_{n-1}{ }^{I} \wedge p_{n}{ }^{l}$, the selection predicate $\Phi_{2}$ is a conjunction of $n+1$ predicates $p_{1}^{2} \wedge p_{2}^{2} \wedge \ldots \wedge p_{n-l^{2}}{ }^{2} \wedge$ $p_{n}{ }^{2}$, for each $1 \leq i \leq(n-1)$ we have $p_{i}{ }^{l}=p_{i}{ }^{2}$, and the predicate $p_{n}{ }^{l}$ is stronger than $p_{n}{ }^{2}$.

(3) There exists a frequent itemset selection predicate $\Phi_{3}$, such that $\Phi_{3} \subset \Phi_{1} \wedge \Phi_{2} \subset \Phi_{3}$.

Proof. The proof is straightforward, based on definitions 3 and 4.

Definition 5 (Restricted data selection predicate). Given are two data mining queries: $d m q_{1}=\left(R_{2} a, \Sigma_{1}, \Phi_{1}, \beta_{1}\right)$ i $d m q_{2}=\left(R_{,} a, \Sigma_{2}, \Phi_{2}, \beta_{2}\right)$. We say that the data selection predicate $\Sigma_{1}$ is restricted with respect to $\Sigma_{2}$ (or $\Sigma_{2}$ is relaxed with respect to $\Sigma_{2}$ ), written as $\Sigma_{2} \subset \Sigma_{1}$, if and only if for each record of $\mathbb{R}_{3}$ satisfying $\Sigma_{1}$ implies also 
satisfying $\Sigma_{2}$. We say that the data selection predicates are independent if $\Sigma_{1} \not \subset \Sigma_{2}$ $\wedge \Sigma_{2} \not \subset \Sigma_{1} \wedge \Sigma_{l} \neq \Sigma_{2}$.

\section{Data Mining Query Execution Using a Materialized Data Mining View}

Let us consider the problem of executing a data mining query using a materialized data mining view. Let $d m q=\left(\mathbb{R}_{l} a, \Sigma_{d m q}, \Phi_{d m q}, \beta_{d m q}\right), d m v_{l}=\left(R_{l} a, \Sigma_{l}, \Phi_{l}, \beta_{l}\right)$. We will discuss different methods of employing $d m v_{l}$ in the process of executing $d m q$. We enumerate six query-view configuration classes, that enable us to use the materialized data mining view: (1) Class I - identical data selection predicates, identical frequent itemset selection predicates, identical minimum supports, (2) Class II - identical data selection predicates, frequent itemset selection predicate relaxed or independent in $d m q$ or minimum support lowered in $d m q$, (3) Class III - identical data selection predicates, frequent itemset selection predicate restricted or equal in $d m q$, minimum support not lowered in $d m q$, (4) Class IV - data selection predicate restricted in $d m v$, identical frequent itemset selection predicates, identical minimum supports, (5) Class $\mathrm{V}$ - data selection predicates restricted in $d m v_{1}$, frequent itemset selection predicate relaxed or independent in $d m q$ or minimum support lowered in $d m q$, (6) Class VI data selection predicate restricted in $d m v_{1}$, frequent itemset selection predicate restricted or equal in $d m q$, minimum support not lowered in $d m q$. Classes I and IV are subclasses of classes III and VI respectively, offering more efficient query answering algorithms. In all other cases (data selection predicates independent or data selection predicate relaxed in $d m q$ ), $d m v_{l}$ is not usable in executing $d m q$ (itemsets contained in $d m v_{I}$ were counted in parts of database that are not relevant to $d m q$ ).

Class I $\left(\Sigma_{l}=\Sigma_{d m q} \wedge \beta_{l}=\beta_{d m q} \wedge \Phi_{l}=\Phi_{d m q}\right)$. Since the materialized data mining view $d m v_{l}$ contains the exact result of the data mining query $d m q$, then the execution of $d m q$ only takes to read the contents of $d m v_{1}$. We will refer to this method as to View Ready $(V R)$. The I/O cost costvR for View Ready involves only the retrieval of $d m v_{1}$ contents:

$$
\text { costvR }=\|d m v i\|,
$$

where $\left\|d m v_{1}\right\|$ is the size of $d m v_{1}$ contents (disk pages).

In order to estimate the benefits of using View Ready, let us consider the I/O cost costFULL of executing a complete frequent itemset discovery algorithm (eg., Apriori) on $\sigma_{\Sigma^{d m q}} R$. The cost involves $k$ scans of $\sigma_{\Sigma d m q} R(k$ depends on the particular algorithm used):

$$
\operatorname{costFULL}=k \cdot\left\|\sigma_{\Sigma^{d m q}} R\right\|,
$$


where $\left\|\sigma_{\text {Zlmq }} R\right\|$ is the I/O cost of retrieving all records of $R$ satisfying $\Sigma_{d m q}$. Notice that View Ready is useful if $\|d m v i\|<k \cdot\left\|\sigma_{2 d m q} R\right\|$. Since in practical application of

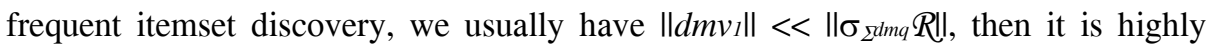
beneficial to use the described method in order to execute a data mining query.

Class II $\left(\Sigma_{l}=\Sigma_{d m q} \wedge\left(\beta_{l}>\beta_{d m q} \vee \Phi_{l} \not \subset \Phi_{d m q}\right)\right)$. Since the materialized data mining view is not guaranteed to contain all itemsets to be returned by $d m q$, the execution of $d m q$ takes to perform a simplified frequent itemset discovery algorithm, eg., Apriori, in which we count only those candidates, that do not belong to $d m v$ ı. If a candidate belongs to $d m v$, then we do not need to count it, because we already know its support. We will refer to this method as to Complementary Mining $(C M)$. The I/O cost costcm for Complementary Mining involves $k$ scans of $\sigma_{Z^{l m} m} R(k$ depends on the particular algorithm used) and a single scan of $d m v$ :

$$
\operatorname{costcM}=k \cdot\left\|\sigma_{\Sigma} d m q R\right\|+\|d m v i\| .
$$

When we compare the I/O cost of Complementary Mining with the I/O cost of executing a complete frequent itemset discovery algorithm (eg., Apriori) on $\sigma_{\Sigma^{l l m}} R$, then we notice that Complementary Mining is more costly compared to not using a materialized data mining view at all. This fact actually eliminates Complementary Mining from practical applications. However, since the I/O cost is only a part of a total cost of executing a data mining query, then in a very specific case it might happen that the I/O overhead gets compensated by an improvement of CPU time. Such effects may occur e.g., in CPU-bound computer systems.

Class III $\left(\Sigma_{l}=\Sigma_{d m q} \wedge \beta_{l} \leq \beta_{d m q} \wedge \Phi_{l} \subseteq \Phi_{d m q}\right)$. Since the materialized data mining view $d m v_{l}$ contains a superset of the result of $d m q$, then the execution of $d m q$ takes to read the contents of $d m v_{l}$ and filter the frequent itemsets with respect to $\beta_{d m q}$ and $\Phi_{d m q}$. We will refer to this method as to Verifying Mining (VM). The I/O cost costvm for Verifying Mining involves only the scan of $d m v t$ :

$$
\text { costvR }=\|d m v i\| .
$$

When we compare the I/O cost of Verifying Mining with the I/O cost of executing a complete frequent itemset discovery algorithm (e.g., Apriori) on $\sigma_{\text {Zdmq }} R$ then we notice that Verifying Mining is useful if $\|d m v i\|<k \cdot\left\|_{\Sigma^{l m m}} R\right\|_{\|}$. According to our discussion above, we conclude that Verifying Mining is highly beneficial.

Class IV $\left(\Sigma_{l} \subset \Sigma_{d m q} \wedge \beta_{l}=\beta_{d m q} \wedge \Phi_{l}=\Phi_{d m q}\right)$. The database has been logically divided into two partitions (1) the records covered by the materialized data mining view $d m v$, (2) the records covered by the data mining query $d m q$, and not covered by the materialized data mining view. Since $d m v_{l}$ contains frequent itemsets discovered only in the first partition, therefore the executing of $d m q$ takes to discover all frequent itemsets in the second partition (eg. using Apriori), to merge the discovered frequent itemsets with the frequent itemsets from $d m v$, and finally to scan the database in 
order to count and filter frequent itemsets. We will refer to this method as to Incremental Mining (IM) since it is similar to inremental updation algorithms. The I/O cost cost the particular algorithm used), a sigle scan of $d m v_{l}$, and a single scan of $\sigma_{\Sigma d m q} \mathbb{R}$ :

$$
\operatorname{costcM}=k \cdot\left\|\sigma_{\left(\Sigma d m q-\left(\Sigma d m q_{\cap} \Sigma^{l}\right)\right.} R\right\|+\|d m v i\|+\left\|\sigma_{\Sigma} d m q R\right\| .
$$

When we compare the I/O cost of Incremental Mining with the I/O cost of executing a complete frequent itemset discovery algorithm (e.g., Apriori) on $\sigma_{\Sigma d m q} R$, then we notice that Incremental Mining is useful if:

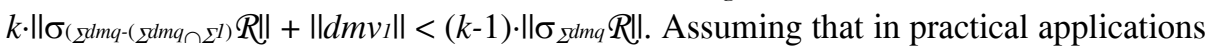
we usually have: $\|d m v i\|<\left\|\sigma_{\left(\Sigma d m q-\left(\Sigma^{d m} q_{\cap} \Sigma^{l}\right)\right.} \mathbb{R}\right\|<\left\|\sigma_{\Sigma^{d} m q} \mathbb{R}\right\|$, it means that Incremental Mining is beneficial (in terms of I/O costs) when $\left\|\sigma_{\left(\Sigma d m q-\left(\Sigma d m q_{\cap} \Sigma^{l}\right)\right.} R\right\|<(k-1) / k \cdot\left\|\sigma_{\Sigma^{d m q}} R\right\|$, which means that e.g., for $k=10$ the materialized data mining view should cover at least $10 \%$ of the dataset covered by the data mining query.

Class $\mathbf{V}\left(\Sigma_{l} \subset \Sigma_{d m q} \wedge\left(\beta_{l}>\beta_{d m q} \vee \Phi_{l} \not \subset \Phi_{d m q}\right)\right)$. The database has been logically divided into two partitions (1) the records covered by the materialized data mining view $d m v$, (2) the records covered by the data mining query $d m q$, and not covered by the materialized data mining view. The materialized data mining view $d m v_{1}$ is not guaranteed to contain all the frequent itemsets that would be discovered in the first partition (using $\beta_{d m q}$ i $\Phi_{d m q}$ ). The execution of $d m q$ is a two-step procedure. In the first step, we execute a simplified frequent itemset discovery algorithm, e.g. Apriori, in which we count only those candidates, that do not belong to $d m v ı$. If a candidate belongs to $d m v i$, then we do not need to count it, because we already know its support. In the second step, we discover all frequent itemsets in the second partition, we merge the discovered frequent itemsets with those from the first step, and finally we scan the database to count and filter them. Formally, this method is a combination of Complementary Mining and Incremental Mining, therefore its I/O cost is the following:

$$
\begin{aligned}
& \operatorname{costcM}+\operatorname{costIM}=k \cdot\left\|\sigma_{\Sigma^{l}} \mathscr{R}\right\|+\left\|d m v v_{l}\right\|+k \cdot\left\|\sigma_{\left(\Sigma^{d m q}-\left(\Sigma^{d m} q_{\cap \Sigma^{l}}\right)\right.} \mathbb{R}\right\|+\left\|\sigma_{\Sigma^{d m q}} \mathscr{R}\right\|=(k+1) \\
& \left\|\sigma_{\Sigma^{d m}} R\right\|+\|d m v \imath\| .
\end{aligned}
$$

When we compare the above I/O cost with the $\mathrm{I} / \mathrm{O}$ cost of executing a complete frequent itemset discovery algorithm on $\sigma_{\Sigma^{d m}} R$, then we notice that in most practical applications the above method is more costly compared to not using a materialized data mining view at all. However, since the I/O cost is only a part of a total cost of executing a data mining query, then in a very specific case it might happen that the I/O overhead gets compensated by an improvement of CPU time. Such effects may occur e.g., in CPU-bound computer systems.

Class VI $\left(\Sigma_{l} \subset \Sigma_{d m q} \wedge \beta_{l} \leq \beta_{d m q} \wedge \Phi_{l} \subseteq \Phi_{d m q}\right)$. The database has been logically divided into two partitions (1) the records covered by the materialized data mining view $d m v$, 
(2) the records covered by the data mining query $d m q$, and not covered by the materialized data mining view. The materialized data mining view $d m v_{l}$ contains a superset of all frequent itemsets that would be discovered in the first partition (using $\beta_{d m q}$ i $\left.\Phi_{d m q}\right)$. The execution of $d m q$ is a two-step procedure. In the first step we scan $d m v_{l}$ and we filter its frequent itemsets with respect to $\beta_{d m q}$ and $\Phi_{d m q}$. In the second step, we discover all frequent itemsets in the second partition, we merge the discovered frequent itemsets with those from the first step, and finally we scan the database to count and filter them. Formally, this method is a combination of Verifying Mining and Incremental Mining, therefore its I/O cost is the following:

$$
\operatorname{costvR}+\operatorname{cost}_{I M}=\|d m v i\|+k \cdot\left\|\sigma_{\left(\Sigma^{d m q}-\left(\Sigma^{d m q} \cap \Sigma^{l}\right)\right.} \mathbb{R}\right\|+\left\|\sigma_{\Sigma^{d m q}} \mathbb{R}\right\| .
$$

When we compare the above $\mathrm{I} / \mathrm{O}$ cost with the cost of executing a complete frequent itemset discovery algorithm on $\sigma_{\Sigma d m q} R$, then we notice that the discussed method is useful if: $\|d m v i\|+k \cdot\left\|\sigma_{\left(\Sigma^{d} m q-\left(\Sigma^{d} d m q_{\cap} \Sigma^{l}\right)\right.} \mathbb{R}\right\|<(k-1) \cdot\left\|\sigma_{\Sigma^{d m q}} \mathbb{R}\right\|$. Assuming that in most

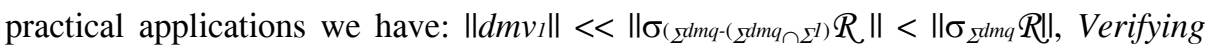
Mining + Incremental Mining is beneficial (in terms of I/O costs) if $\left\|\sigma_{\left(\Sigma d m q-\left(\Sigma d m q_{\cap} \Sigma^{l}\right)\right.} R\right\|$ $<(k-1) / k \cdot\left\|\sigma_{\Sigma^{d m q}} R\right\|$. For instance, for $k=10$ it means that the materialized data mining view should cover at leas $10 \%$ of the dataset covered by the data mining query.

Our above discussion has been summarized in the Table 2.

Table 2. Methods of executing a data mining query using a materialized data mining view

\begin{tabular}{|c|c|c|}
\cline { 2 - 3 } \multicolumn{1}{c|}{} & $\Sigma_{l}=\Sigma_{d m q}$ & $\Sigma_{l} \subset \Sigma_{d m q}$ \\
\hline$\beta_{l}=\beta_{d m q} \wedge \Phi_{l}=\Phi_{d m q}$ & $V R$ & $I M$ \\
\hline$\beta_{l}>\beta_{d m q} \vee \Phi_{l} \not \subset \Phi_{d m q}$ & $C M$ & $C M, I M$ \\
\hline$\beta_{l} \leq \beta_{d m q} \wedge \Phi_{l} \subseteq \Phi_{d m q}$ & $V M$ & $V M, I M$ \\
\hline
\end{tabular}

\section{Experimental Results}

In order to evaluate performance gains thanks to using a materialized view, we performed several experiments on a Pentium II 433MHz PC with $128 \mathrm{MB}$ of RAM. We experimented with synthetic and real datasets. The synthetic datasets were generated by means of the GEN generator from the Quest project [2]. The real datasets that we have used come from the UCI KDD Archive [6]. Here we report results on the MSWeb $^{1}$ (Microsoft Anonymous Web Data) dataset and a synthetic dataset containing 148000 transactions built from 100 different items, with the average transaction size of 30 .

In the tests we did not consider Class I (trivial, in practice always beneficial) and classes involving Complementary Mining, i.e., II and V (theoretically proven as inefficient). Thus, we focused on practical verification of Verifying Mining and

\footnotetext{
${ }^{1}$ http://kdd.ics.uci.edu/databases/msweb/msweb.html
} 
Incremental Mining. As a complete data mining algorithm we used our implementation of Apriori. To simulate constraints of a multi-user environment, we limited the amount of main memory available to algorithms to $10-50 \mathrm{kB}$. Each of the charts presents average results from a series of 20 experiments.

In the first series of experiments we varied the level of coverage of the query's dataset by materialized view's dataset. The minimum support of the query was by $10 \%$ higher than in case of the materialized view. Figure 2 presents the results for real and synthetic datasets.
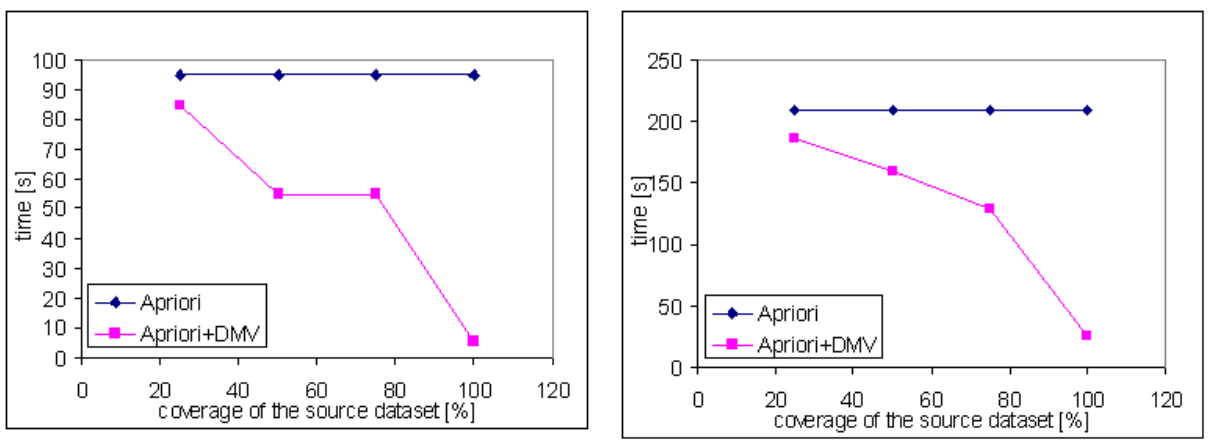

Fig. 2. Execution times for various levels of coverage of the query's dataset by materialized view's dataset for real (left) and synthetic (right) datasets

The experiments show that even for a materialized view based on the dataset covering $20 \%$ of the query's dataset, exploiting the results stored in the view reduces processing time. In general, more significant coverage results in better performance of the method using a materialized view. However, the exact performance improvement depends also on data distribution and the support threshold.

In the second series of experiments we tested the impact of difference between the support thresholds of the query to be answered and the materialized data mining view. The results for both considered datasets are presented in Fig. 3. The difference between the thresholds is expressed as the percentage of the support threshold of the query to be answered (the support threshold used for the materialized view was lower than the support threshold used in the query). For both datasets the source dataset for the view covered $75 \%$ of the dataset of the query. 

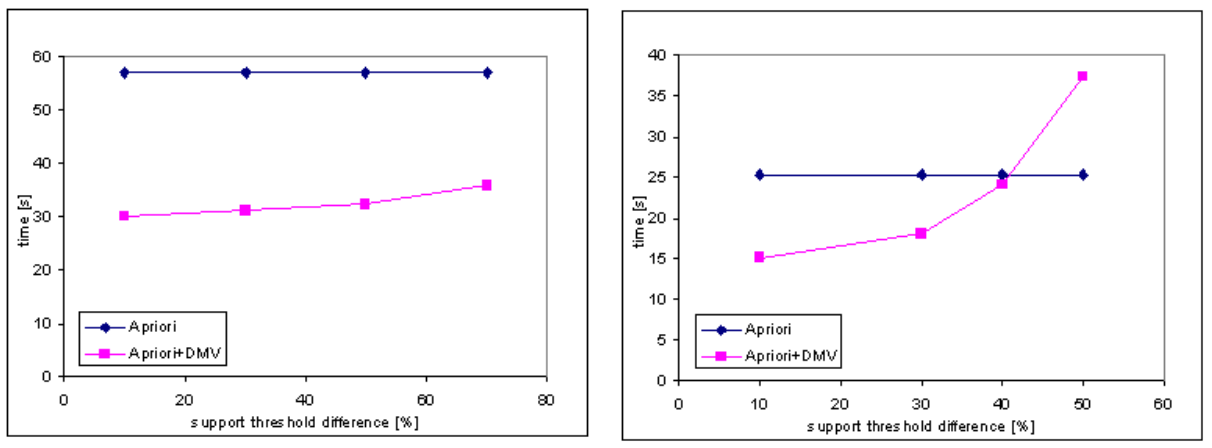

Fig. 3. Execution times for various relative differences in support thresholds for real (left) and synthetic (right) datasets

The experiments show that using a materialized view is more efficient when the difference between the support threshold is small. For big differences it is even possible that using a materialized view is a worse solution than running the complete frequent itemset mining algorithm. This can happen since for a very low support threshold the size of a materialized view can be very big, introducing high I/O costs. The exact value of the difference between support thresholds for which using a materialized view is not beneficial depends on the actual threshold values, the nature of the dataset, and the coverage of the query's dataset by materialized view's dataset.

\section{Conclusions}

In this paper we discussed answering a frequent itemset query using a materialized data mining view. We classified possible differences between the current query and the query defining the materialized view into six classes. We provided I/O cost analysis for all the classes, and experimentally evaluated the most promising ones.

Theoretical analysis and experiments show that using a materialized view is an efficient solution in cases for which View Ready, Verifying Mining, and Incremental Mining techniques are applicable. The latter two methods perform particularly well when the support threshold of the view is close to the support threshold of the query and/or the source dataset of the view covers significant part of the query's dataset.

In the future we plan to consider situations in which a given query can be answered using a collection of materialized data mining views.

\section{References}

1. Agrawal R., Imielinski T., Swami A.: Mining Association Rules Between Sets of Items in Large Databases. Proc. of the 1993 ACM SIGMOD Conf. on Management of Data (1993) 
2. Agrawal R., Mehta M., Shafer J., Srikant R., Arning A., Bollinger T.: The Quest Data Mining System. Proc. of the 2nd Int'l Conference on Knowledge Discovery in Databases and Data Mining, Portland, Oregon (1996)

3. Agrawal R., Srikant R.: Fast Algorithms for Mining Association Rules. Proc. of the 20th Int'l Conf. on Very Large Data Bases (1994)

4. Baralis E., Psaila G.: Incremental Refinement of Mining Queries. Proceedings of the 1st DaWaK Conference (1999)

5. Cheung D.W.-L., Han J., Ng V., Wong C.Y.: Maintenance of discovered association rules in large databases: An incremental updating technique. Proc. of the 12th ICDE Conference (1996)

6. Hettich S., Bay S. D.: The UCI KDD Archive [http://kdd.ics.uci.edu]. Irvine, CA: University of California, Department of Information and Computer Science (1999)

7. Imielinski T., Mannila H.: A Database Perspective on Knowledge Discovery. Communications of the ACM, Vol. 39, No. 11 (1996)

8. Morzy T., Wojciechowski M., Zakrzewicz M.: Materialized Data Mining Views. Proceedings of the 4th PKDD Conference (2000)

9. Morzy T., Wojciechowski M., Zakrzewicz M.: Fast Discovery of Sequential Patterns Using Materialized Data Mining Views. Proceedings of the 15th ISCIS Conference (2000)

10.Nag B., Deshpande P.M., DeWitt D.J.: Using a Knowledge Cache for Interactive Discovery of Association Rules. Proceedings of the 5th KDD Conference (1999)

11.Roussopoulos N.: Materialized Views and Data Warehouses. SIGMOD Record, 27(1) (1998) 